\section{WEB WATCH}

Garnering resources

The Arabidopsis community

has recently acquired a comprehensive genomic

Web-based resource called

the Genomic Arabidopsis

Resource Network (GARNet).

The GARNet Web site can be best described as a portal to access and consolidate various distributed resources that are being created as a result of the GARNet initiative.

The site was established as part of the BBSRC

Investigating Gene Function initiative and aims to create widely accessible functional genomics facilities for the Arabidopsis community. GARNet promises various resources and services, including transcriptome analysis, metabolite profiling, proteome analysis,

bioinformatics, and forward and reverse genetics. Most of these facilities are already available to individual researchers and can be applied for directly from the GARNet home page; a transcriptome analysis resource is planned for early next year. These services are provided and maintained by GARNet service providers individual research groups located in the United Kingdom.

Site visitors will not actually find any data to browse at GARNet itself because data generated from GARNet resources are distributed through the Nottingham Arabidopsis Stock Centre and John Innes Centre databases.

The progress of the project will be monitored by the Garnet Steering Committee, and reviewed at annual open meetings (the next one is on September 27-28, 2001). The Committee plans more regular updates on GARNet developments by producing a news bulletin, which will be distributed to ARAB-UK network subscribers. At present, updates on resource availability can be obtained from GARNish - the GARNet Gazette, which is accessible from the GARNet home page.

Magdalena Skipper

\title{
One very tough bug
}

Deinococcus radiodurans (DEIRA) - literally 'strange berry that withstands radiation' - first came to the attention of scientists when it refused to die in food sterilization tests. It was noted that this bacterium is highly resistant to many extreme conditions, including insults from genotoxic chemicals, high levels of ionizing and ultraviolet radiation, and dehydration. DEIRA is so resilient to DNA damage that it can survive a dose of ionizing radiation 3,000 times higher than it would take to kill a human. Heat, dehydration and radiation normally kill cells by causing double-stranded breaks in their DNA one of the most difficult kinds of DNA damage to repair. It's not that DEIRA can shield its genome from the radiation; but remarkably it can, within hours, stitch back together the hundreds of fragments of broken DNA that result — an outstanding feat, even by prokaryotic standards. Not surprisingly, discovering the secret behind DEIRA's efficient DNA-repair talents has been the focus of many studies. Now, a report by Karlin and Mrázek suggests that DEIRA gets its diehard qualities by producing a protected cellular environment in which normal repair can occur undisturbed.

Genome repair in DEIRA was initially proposed to involve a twostep process: first, broken DNA fragments would be reconnected by annealing free single strands; second, the damaged site would be replaced by homologous recombination. The RecA protein was proposed to speed up recombination by taking advantage of DEIRA's multiple (4-10) genome complements, which, by stacking up like doughnuts on top of each other, lend their homologous segments to the repair machinery. However, the above processes and the presence of additional genome complements are not unique to DEIRA — they are typical of other prokaryotes as well.

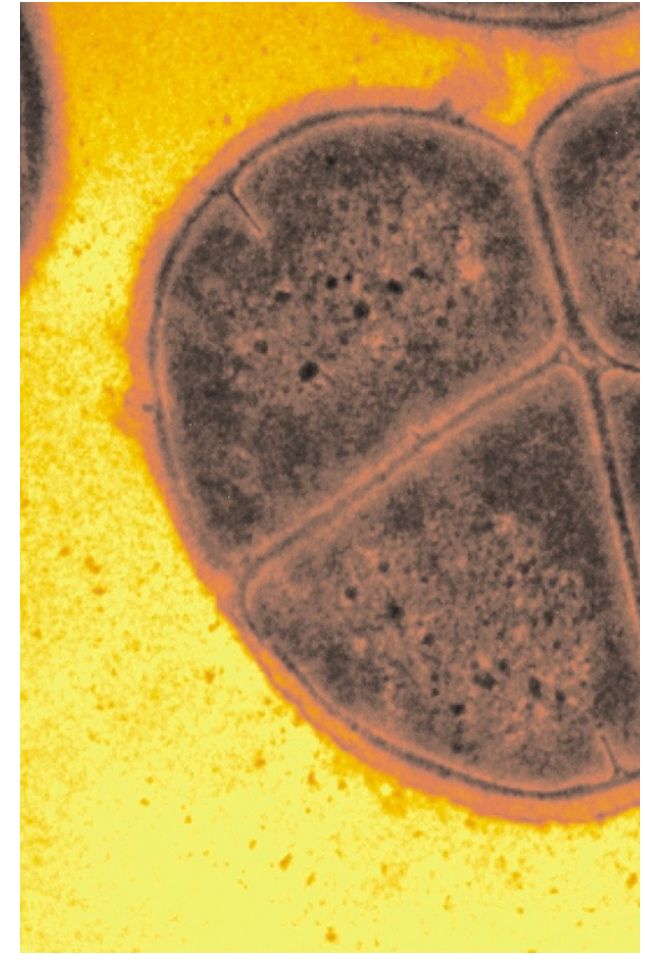

Courtesy of A. Vasilenko and M. Daly, Uniformed Bethesda, Maryland, USA.

For more clues to this mystery, Karlin and Mrázek turned to the DEIRA genome itself - using the codon preference of individual genes to predict their expression levels. Genes that deviate in codon usage from the average gene but that are similar in codon usage to ribosomal proteins (which are expressed at high levels), are also predicted to be highly expressed (PHX). Karlin and Mrázek propose

\section{A nonsensical quest}

Around one-third of all disease mutations in humans cause premature termination of translation. So if there was a way to find transcripts in a cell that carry such mutations, then that would be a powerful tool for diagnosing the genetic cause of any disease. Noensie and Dietz have devised an elegant method that is close to achieving this.

Cells normally degrade any message that contains a premature termination codon, introduced for example by a nonsense or frameshift mutation. This surveillance mechanism - nonsense-mediated mRNA decay (NMD) - is thought to protect cells from the potentially deleterious effects of truncated proteins. Noensie and Dietz reasoned that inhibition of NMD would therefore increase the abundance of transcripts that contain premature termination codons.

NMD is a response to stalled ribosomes and so is dependent on translation. To inhibit NMD, the authors therefore used inhibitors of translation. Not surprisingly, the inhibitors affect the level of some transcripts whether or not they contain a nonsense mutation, but this background can be reduced by judicious use of controls. Microarrays are then used to detect transcripts the rise in abundance of which is caused by inhibition of NMD rather than by some other effect of the inhibitor.

The method was validated in two cell lines, each carrying a single nonsense mutation. Around 5,000 genes were screened, and a list of genes that increase in abundance in the presence of the inhibitor was obtained for each line. The target genes were 19th and 48th on the two lists - so the method isn't perfect. However, when you consider the location of the genes, or information about their function, the target genes stand out as much stronger candidates. 